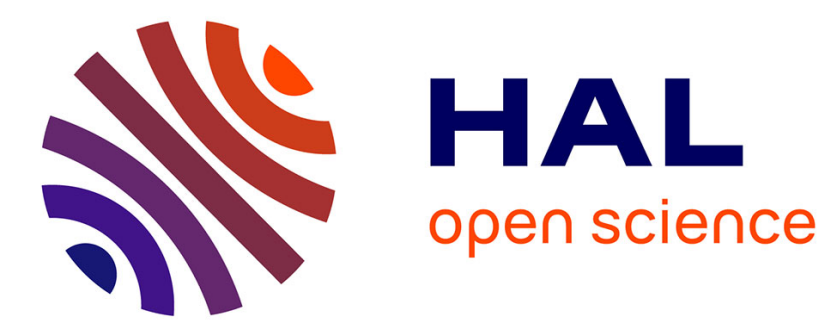

\title{
Quelques constats sur les prévisions conjoncturelles de la croissance française
}

\author{
Thomas Jobert, Lionel Persyn
}

\section{To cite this version:}

Thomas Jobert, Lionel Persyn. Quelques constats sur les prévisions conjoncturelles de la croissance française. 2011. halshs-00721673

\section{HAL Id: halshs-00721673 \\ https://shs.hal.science/halshs-00721673}

Preprint submitted on 29 Jul 2012

HAL is a multi-disciplinary open access archive for the deposit and dissemination of scientific research documents, whether they are published or not. The documents may come from teaching and research institutions in France or abroad, or from public or private research centers.
L'archive ouverte pluridisciplinaire HAL, est destinée au dépôt et à la diffusion de documents scientifiques de niveau recherche, publiés ou non, émanant des établissements d'enseignement et de recherche français ou étrangers, des laboratoires publics ou privés. 


\title{
Quelques constats sur les prévisions conjoncturelles de la croissance française \\ (Décembre 2011)
}

Thomas Jobert : Auteur correspondant, GREDEG, UMR de l'Université de Nice Sophia Antipolis et du CNRS (UMR 6227).

Université Nice Sophia Antipolis, 24 Avenue des Diables Bleus, 06357 Nice Cedex 4.

e-mail: thomas.jobert@unice.fr

Lionel Persyn : Université de Nice Sophia Antipolis, 24 Avenue des Diables Bleus, 06357

Nice Cedex 4. e-mail: lionel.persyn@unice.fr

\section{Résumé :}

Nous étudions la qualité des prévisions de croissance pour la France réalisées durant la première décennie de ce siècle. Un biais d'optimisme des prévisions Consensus de la croissance trimestrielle apparaît dès l'examen graphique, et son existence est confirmée par le calcul des moyennes des erreurs de prévisions qui sont systématiquement négatives. L'indicateur de Theil montre que les prévisions de l'Insee dominent celles du Consensus qui obtiennent de meilleurs résultats qu'une prévision naïve (donnée par le dernier taux de croissance connu). Les tests semblent confirmer la présence d'un biais d'optimisme dans les prévisions trimestrielles, biais qui peut provenir dans le cas des prévisions Consensus de l'agrégation des prévisions collectées par le Consensus Economics.

L'étude des prévisions de croissance pour l'année à venir faites par chaque organisme interrogé mensuellement par le Consensus Economics nécessite un traitement préalable à cause des données manquantes. Au final nous ne retenons que les prévisions de neuf institutions que nous comparons avec celles du gouvernement, du FMI, de l'OCDE, et de la Commission Européenne. Il apparait d'une part que les prévisions sont très proches de la prévision Consensus, et d'autre part que le biais d'optimisme reste présent. Enfin, le désaccord entre les institutions s'accroît à l'approche d'une phase de contraction et décroît après.

JEL classification : E 37, C 52, C 53.

Mots clefs : Prévisions macroéconomiques ; Evaluation des prévisions ; Rationalité ; Prévisions roulantes ; Prévisions sur événement fixé ; France.

\begin{abstract}
:
We study the quality of the French growth forecasts from the first decade of the century. An optimism bias of the quarterly Consensus growth forecasts can be asserted right from a graphic analysis and its existence is confirmed by the calculation of the mean errors which are systematically negative. The Theil index reveals that Insee forecasts are superior to the Consensus ones. In addition, the Consensus forecasts are superior to a naïve forecast. Proper tests seem to confirm that an optimism bias exists ; this bias could arise from the combination of several forecasts.

The study of the Consensus Economics fixed event forecasts regarding the next coming year requires a preliminary analysis due to missing data. We thus only retain the forecasts of nine institutions that we compare to those of the Government, the IMF, the OECD, and the European Commission. It appears that the forecasts are fairly close to the Consensus forecast and that the optimism bias is still observable. Finally, the disagreement between the forecasters increases towards a recession and, then, decreases.
\end{abstract}

Some evidences about French growth forecasts

Keywords : Macroeconomic Forecasting ; Evaluating Forecast ; Rationality ; Rolling Event Forecasts ; Fixed Event Forecast ; France 


\section{Introduction}

La crise de 2008 a mis en lumière une accélération du temps économique imposant une dictature de l'immédiateté. Dorénavant les décisions économiques doivent être prises très rapidement, jusqu'à se soumettre à l'urgence, alors que la globalisation financière, la spéculation généralisée, l'intégration régionale, la fragmentation des processus de production, et toutes les autres conséquences de la mondialisation ont énormément complexifié la compréhension du fonctionnement de l'économie d'un pays.

C'est dans ce contexte qu'il est demandé aux économistes de fournir des prévisions chiffrées portant sur l'évolution future des grands agrégats macroéconomiques ${ }^{1}$. La plus attendue est sans aucun doute celle du PIB car cet agrégat permet de mesurer le taux de croissance d'un pays. De plus, comme de nombreux ratios sont rapportés à la richesse produite dans le pays (entre autres les critères de convergence de Maastricht sur les finances publiques), leurs prévisions sont directement liées à celles du PIB. Rappelons enfin que la LOLF donne obligation au gouvernement de présenter avec le projet de loi de finances un rapport précisant notamment les prévisions de croissance retenues pour les quatre années à venir.

Il existe donc aujourd'hui une demande très forte pour une prévision de la croissance qui soit la plus fiable possible. D'un côté les outils utilisés sont beaucoup plus sophistiqués mais, comme la compréhension de l'environnement économique est beaucoup plus complexe, la prévision est rendue plus délicate. L'erreur de prévision est inhérente à tout exercice de prospective et la littérature regorge de travaux sur la qualité des prévisions ${ }^{2}$. La grande majorité (comme par exemple Patton et Timmermann [2011]) se concentre sur la prévision moyenne (appelée aussi prévision consensus) émanant d'un ensemble d'experts travaillant sur l'économie américaine. Les exceptions portent sur des études menées soit à partir de données désagrégées (institution par institution), soit sur des pays autres que les Etats-Unis. Ces dernières sont beaucoup plus rares notamment car les prévisions collectées par le Consensus Economics ${ }^{3}$ ont été relativement peu utilisées bien que cela tende à s'inverser depuis les dix dernières années. Nous n'avons recensé que six études récentes comprenant, entre autres, des données concernant la croissance française. Timmermann [2007] s'intéresse surtout aux prévisions du FMI pour un grand ensemble de pays. Isiklar, Lahiri et Loungani [2006], Ager,

\footnotetext{
${ }^{1}$ Cet exercice périlleux ne consiste pas seulement à déterminer quelques chiffres clefs, il faut aussi qu'ils s'inscrivent dans un scénario, une argumentation qui soutient une logique et une histoire.

${ }^{2}$ L'idée fondamentale sur laquelle se fondent ces travaux est que l'erreur de prévision doit être un bruit blanc.

${ }^{3}$ Consensus Economics existe depuis 1989. Sa publication "Consensus Forecasts" recense chaque mois les prévisions de diverses institutions (une vingtaine pour la France) pour de nombreux pays.
} 
Kappler et Osterloh [2009] et Dovern et Weisser [2011] utilisent une approche par données de panel et l'appliquent aux prévisions du Consensus Economics relatives à divers pays développés. Loungani, Steckler et Tamirisa [2009] et Dovern, Fritsche et Slacalek [2009] étudient l'information apportée par le désaccord entre les analystes sur un grand nombre de pays.

Les travaux que nous venons de citer se focalisent souvent sur l'utilisation de techniques statistiques permettant d'étudier la qualité des prévisions sans toujours s'interroger sur la nature de l'objet analysé. Une série chronologique de prévisions provient d'un exercice de prospective mobilisant d'abord un espace d'informations le plus précoce et le plus précis possible. Or, cet espace n'est pas invariant dans le temps et s'enrichit constamment soit grâce à l'apport de nouvelles enquêtes ou de nouveaux indices quantitatifs, soit grâce à l'apparition de nouveaux indicateurs synthétiques (climat des affaires, retournement, etc.). Ensuite, les outils utilisés pour mener la prévision peuvent évoluer même si une prévision est le plus souvent élaborée par une combinaison de méthodes très différentes qui vont de la simple intuition (les dires d'experts) aux modèles macroéconométriques, en passant par les équations d'étalonnages et les modèles en boite noire. Ces derniers modèles qualifiés aussi de modèles a-théoriques (modèles VAR, VECM, modèles à facteurs ${ }^{4}$ ) prolongent la tendance tout en utilisant au mieux toute l'information conjoncturelle disponible. Enfin, l'erreur de prévision est calculée en référence à une "réalité" dont la mesure évolue au gré des modifications de la comptabilité nationale, et dont l'évaluation de la "réalisation" varie au gré des révisions effectuées entre les comptes provisoires et les comptes définitifs ${ }^{5}$. Pour mémoire rappelons que la première estimation du PIB d'un trimestre est donnée 42 jours après la fin du dit trimestre, qu'il faut attendre la mi-février de l'année N+1 pour avoir une première estimation du PIB de l'année N, et que les comptes définitifs de l'année $\mathrm{N}$ ne sont connus qu'en mai de l'année $\mathrm{N}+3$. Ainsi, la complexité intrinsèque de la notion d'erreur de prévision nécessite de faire un arbitrage dans la construction de la base de données entre la profondeur des données et l'homogénéité de l'objet étudié, choix sur lesquels nous reviendrons par la suite.

Les prévisions de l'évolution du PIB français rendues publiques émanent aussi bien d'organismes internationaux ou nationaux, que d'institutions privées. Le FMI (World Economic Outlook), l'OCDE (Perspectives économiques de l'OCDE), et la Commission

\footnotetext{
${ }^{4}$ F. Charpin (2011) compare les performances de ces modèles alors que Bessec (2010) propose un travail original sur la construction d'équations d'étalonnage du PIB français.

${ }^{5}$ J-M Charpin, directeur général de l'Insee entre 2003 et 2007, reconnaît que la qualité de l'information délivrée par les comptes nationaux provisoires fait débat. " Chaque publication peut relancer les critiques sur la volatilité des estimations et l'ampleur des révisions, qui sont d'ailleurs compréhensibles compte tenu de l'importance de ces statistiques pour le diagnostic économique" [Charpin (2010), p 378].
} 
Européenne (European Economic Forecast) publient deux fois par an des prévisions de croissance pour l'année en cours et pour l'année à venir. L'Insee (notes et points de conjoncture) diffuse chaque trimestre depuis mars 1997 sa prévision de croissance pour le trimestre en cours et le trimestre à venir. La seule prévision du gouvernement à s'inscrire dans un calendrier régulier est celle donnée chaque mois de septembre dans le projet de loi de finance. Dans le domaine de la collecte et de la diffusion de prévisions issues des institutions autres que les grands organismes nationaux ou internationaux Consensus Economics est le leader mondial. Sa publication mensuelle "Consensus Forecasts" propose deux types de prévisions de croissance : d'une part une prévision de chaque institution interrogée pour l'année en cours et pour l'année à venir, d'autre part la prévision trimestrielle moyenne (Quarterly Consensus Forecasts) pour le trimestre courant et pour les six trimestres à venir.

Nous disposons donc de deux sortes de prévisions de la croissance conjoncturelle, une qui porte sur les évolutions trimestrielles du PIB, l'autre sur les prévisions annuelles. Les données les plus récentes sont celles de l'Insee qui n'existent dans une fréquence trimestrielle que depuis mars 1997. Cependant, pour des raisons d'homogénéité que nous discuterons dans la section 2, nous n'étudierons que les prévisions émises à partir de 1999. Comme les comptes définitifs ne sont connus qu'en $\mathrm{N}+3$ et pour ne pas perdre de données, nous mesurerons l'erreur de prévision en référence à la première estimation provisoire du PIB, ce qui nous permet d'aller jusqu'en 2010.

L'objectif de notre article est de proposer un panorama le plus complet possible des prévisions conjoncturelles de la croissance française sur la première décennie du vingt-et-unième siècle et d'en analyser les propriétés. Une question centrale sera de voir si la multiplication des outils permettant de mener des prévisions et l'abondance des institutions proposant des prévisions permettent d'améliorer la qualité de l'information ou au contraire si elles créent un brouillard. La première section sera consacrée à l'analyse des prévisions trimestrielles soit au moyen d'indicateurs de qualité, soit à partir de tests. Après avoir discuté de la possibilité d'un biais d'agrégation, nous étudions dans la seconde section les prévisions individuelles des organismes interrogés par le Consensus Economics. Après avoir comparé ces prévisions à celles issues d'organisations internationales ou nationales, nous en testerons les propriétés.

\section{Les prévisions roulantes (rolling event forecasts) ${ }^{6}$}

Ces prévisions sont publiées à un rythme trimestriel (mars, juin, octobre, et décembre) par l'Insee et par le Consensus Economics. Les plus précoces de quelques jours sont les prévisions agrégées (par une simple moyenne) de la vingtaine d'organismes interrogés par le Consensus

\footnotetext{
${ }^{6}$ L'écart entre la date d'émission de la prévision et la cible prévue ne dépend que de l'horizon considéré.
} 
Economics qui concernent le trimestre courant et les six trimestres à venir. De son coté l'Insee propose dans sa note de conjoncture des prévisions portant sur le trimestre courant et sur le trimestre à venir. En juin et décembre, ces prévisions sont étendues à deux trimestres.

\section{I.1. Un premier regard sur les données}

La période retenue porte sur des prévisions émises entre 1999 et 2009. La cible prévue est le taux de croissance trimestriel en glissement annuel à des horizons allant du trimestre courant à six trimestres.

\section{Graphique 1 : La croissance en glissement annuel et les prévisions} Consensus

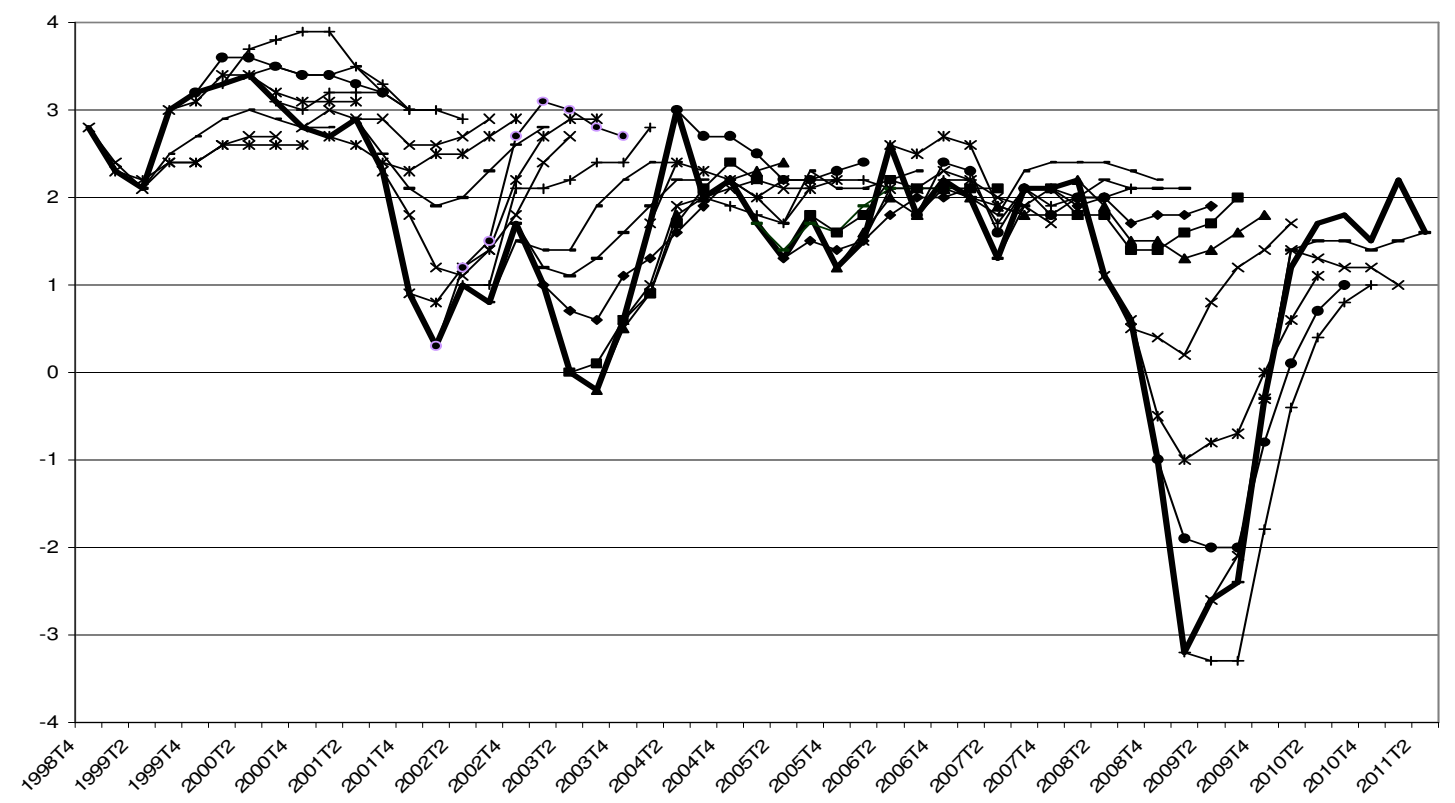

Source: Consensus Economics.

La courbe en gras représente la cible à prévoir, le taux de croissance du PIB calculé ici en glissement annuel. De celle-ci partent des courbes en trait fin qui représentent les prévisions Consensus à un horizon de sept pas (pour le trimestre courant et les six trimestres à venir). L'erreur de prévision est mesurée par la distance entre la courbe en gras et les courbes en trait fin.

Pour un problème de lisibilité du graphique 1 nous ne pouvons reporter les prévisions de l'Insee à côté de celles du Consensus. Un premier examen rapide du graphique 1 révèle que les prévisions (les courbes en trait fin) sont très majoritairement au dessus de la réalisation (la courbe en trait gras), ce qui est caractéristique d'un biais d'optimisme, et que les erreurs de prévision les plus importantes surviennent durant les crises. Un examen plus approfondi se focalisant sur les deux crises montre que le ralentissement de 2001 et la reprise de l'été 2002 sont assez bien anticipés, tant dans leur ampleur que dans leur durée. En revanche comme les conjoncturistes s'attendaient à une crise en U (et non à une crise en W) les prévisions pour 2002-2003 sont très mauvaises. Pour 2008 le ralentissement est bien anticipé mais son 
ampleur est très largement sous-estimée. Quant à la reprise de 2009, les prévisions du premier trimestre font état d'une stabilisation de la récession, l'opinion générale étant qu'il va falloir attendre 2010 pour voir la situation s'améliorer. Or, la reprise sera beaucoup plus précoce et plus dynamique.

Tableau 1 : Quelques statistiques sur les erreurs de prévision

\begin{tabular}{|l|l|c|c|c|c|c|c|c|}
\hline & & \multicolumn{7}{|c|}{ Horizon en trimestre } \\
\hline & & 0 & 1 & 2 & 3 & 4 & 5 & 6 \\
\hline \multirow{3}{*}{ Insee } & Moyenne & -0.091 & -0.184 & - & - & - & - & - \\
\cline { 2 - 10 } & RMSE & 0.374 & 0.611 & & - & - & - & - \\
\hline \multirow{3}{*}{ Consensus } & Moyenne & -0.136 & -0.284 & -0.445 & -0.625 & -0.773 & -0.939 & -1.023 \\
\cline { 2 - 9 } & RMSE & 0.450 & 0.694 & 1.039 & 1.383 & 1.633 & 1.746 & 1.839 \\
\hline
\end{tabular}

Le tableau 1 propose plusieurs statistiques sur les erreurs de prévision. L'erreur moyenne $\left(\frac{1}{N} \sum_{i}\left(R_{i}-P_{i}\right)\right.$ où $\mathrm{N}$ est le nombre total de prévisions au même horizon, $R_{i}$ la réalisation et $P_{i}$ la prévision) mesure tout simplement le biais moyen des prévisions. Un indicateur de la précision absolue de la prévision est obtenu en mesurant la variance des points autour de la ligne de parfaite prévision. C'est la $R M S E=\sqrt{\frac{1}{N} \sum_{i}\left(R_{i}-P_{i}\right)^{2}}$.

Les enseignements du tableau 1 sont triples. Premièrement les moyennes des erreurs de prévision sont systématiquement négatives, ce qui confirme la thèse d'un biais d'optimisme. Deuxièmement les prévisions de l'Insee sont systématiquement meilleures (aussi bien en moyenne qu'en précision) que celles du Consensus Economics. Ce constat n'est pas étonnant puisque d'une part les prévisions du Consensus Economics sont plus précoces que celles de l'Insee et que d'autre part l'institut national statistique dispose à court terme d'un espace d'information bien meilleur que les institutions privées. Troisièmement nous retrouvons un résultat attendu dans le cas où la fonction de perte des organismes est quadratique : la valeur absolue de l'erreur de prévision croit avec l'horizon et sa précision décroit (Voir Granger [1969]) .

\section{2. Propriétés des prévisions}

Un moyen simple de comparer des prévisions obtenues par deux méthodes différentes est donné par l'indicateur de Theil $\left(\frac{R M S E}{R M S E \text { Méthode alternative }}\right)$. Si le ratio est inférieur à l'unité la méthode alternative donne de moins bonnes prévisions. Les résultats ${ }^{7}$ de la

\footnotetext{
${ }^{7}$ Nous ne pouvons pas reporter l'ensemble des résultats qui sont disponibles sur demande auprès des auteurs.
} 
comparaison des prévisions Consensus à celles de l'Insee pour le trimestre courant et le trimestre à venir indiquent que les prévisions de l'Insee dominent celles du consensus, mais que l'écart tend à disparaître en fin de période.

\section{Graphique 2 : Décomposition de l'indicateur de Theil pour les prévisions Consensus (en comparaison avec une prédiction naïve)}

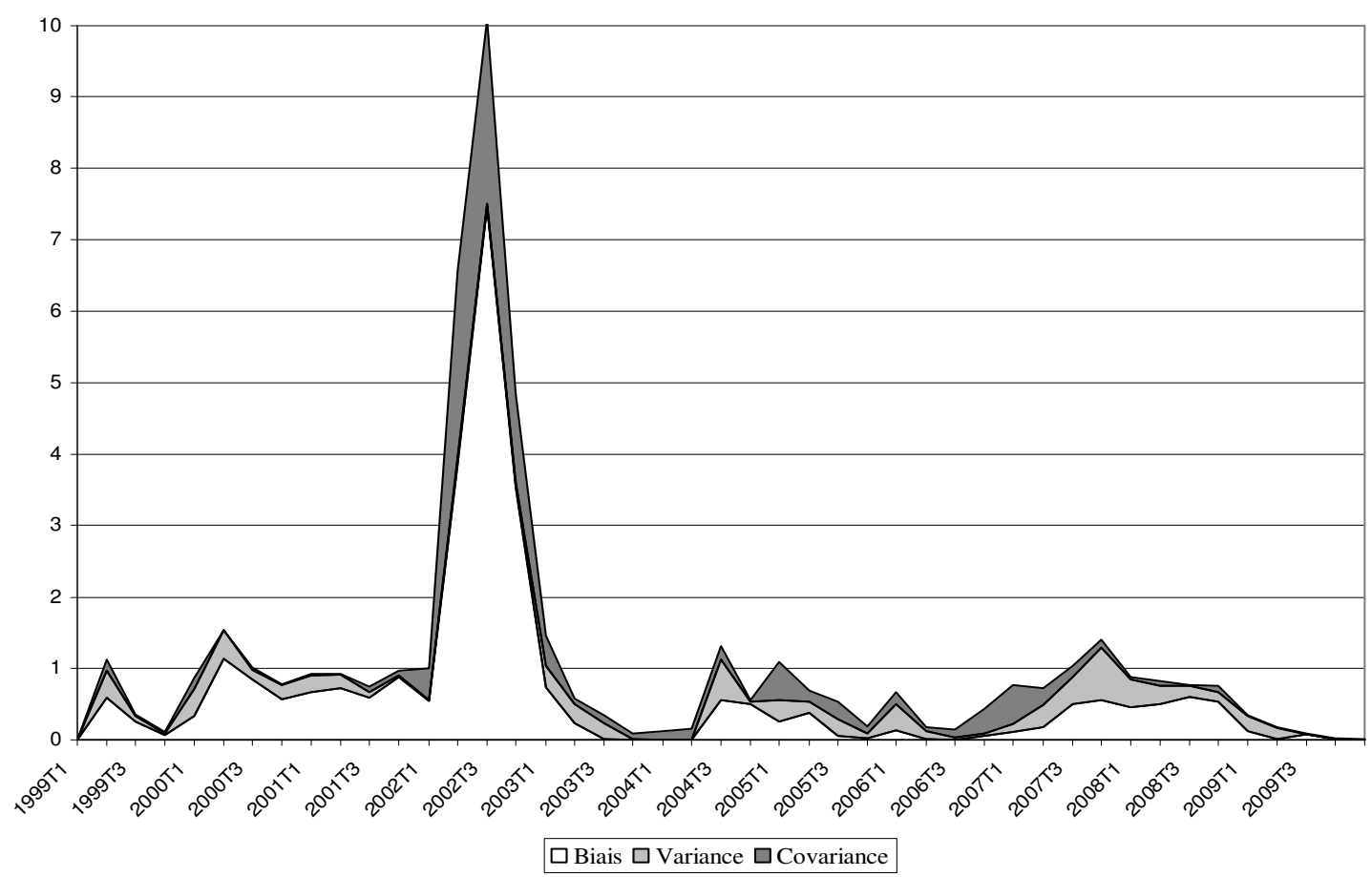

Un autre exercice consiste à se focaliser sur les prévisions Consensus et à les comparer avec celles issues d'une prédiction naïve (le taux de croissance prévu est égal au dernier taux de croissance observé). Nous avons de plus décomposé la RMSE du consensus en trois composantes : l'erreur de tendance centrale (le biais dû à la mauvaise estimation de la moyenne de la variable à prévoir), la valeur de la variation (la variance), et la covariance (qui n'existe qu'en cas d'imparfaite corrélation entre les prévisions et les réalisations). Les résultats présentés sur le graphique 2 permettent de tirer trois constats : les prévisions Consensus dominent globalement les prédictions naïves, sauf durant le second retournement de la crise de 2001-2003. La composante du biais est en moyenne majoritaire mais elle tend à diminuer en fin de période. La crise de 2008 a été mieux prévue qu'elle ne l'aurait été par une prévision naïve.

La littérature foisonne d'articles sur les notions de rationalité et d'efficience des prévisions, ainsi que sur les méthodes les plus adéquates pour les tester ${ }^{8}$. Dans le cas de prévisions roulantes, la démarche de Mincer et Zarnowitz [1969] demeure la plus populaire pour vérifier

\footnotetext{
${ }^{8}$ Se référer à Pesaran et Weale [2006] pour une large revue de la littérature.
} 
la rationalité et a fait l'objet de nombreuses améliorations ${ }^{9}$. Elle repose sur l'équation $R_{t}=a+b P_{t}+u_{t}$ où $R_{i}$ est la réalisation et $P_{i}$ la prévision. Les tests de rationalité des prévisions reposent sur le test de l'hypothèse : $(\mathrm{a}=0, \mathrm{~b}=1)$. Cependant comme les résidus peuvent être autocorrélés et que le taux de croissance est inconnu en temps réel, un test de Fisher à partir des estimateurs des MCO peut être erroné et il convient d'utiliser la Méthode des Moments Généralisés (GMM). Ceci n'est valable que si les séries sont stationnaires, ce que nous avons vérifié, aussi bien sous l'hypothèse nulle (KPSS) que sous l'hypothèse alternative (ADF).

\section{Tableau 2 : P. Value associé aux tests}

\begin{tabular}{|l|l|c|c|c|c|c|c|c|}
\hline \multirow{2}{*}{ Test de rationalité } & \multicolumn{6}{|c|}{ Horizon (en trimestre) des erreurs de prévisions } \\
\cline { 3 - 10 } & Courant & 1 & 2 & 3 & 4 & 5 & 6 \\
\cline { 2 - 9 } & Consee & $\mathbf{0 . 0 5}$ & 0.004 & - & - & - & - & - \\
\hline \multirow{2}{*}{ Test de biais } & Insee & 0.04 & 0.04 & 0.03 & 0.01 & 0.02 & 0.008 & 0.0005 \\
\cline { 2 - 9 } & Consensus & 0.029 & 0.012 & 0.018 & 0.010 & 0.006 & 0.0004 & 0.0003 \\
\hline
\end{tabular}

Le tableau 2 donne la $\mathrm{P}$. Value (la probabilité de rejeter à tort l'hypothèse nulle) du test de Mincer et Zarnowitz [1969], et du test de biais appliqué par Timmerman [2007]. Si la P. Value est supérieure à un certain seuil (par exemple 5\%) l'hypothèse nulle (rationalité ou sans-biais) est rejetée. Ce tableau montre que les seules prévisions dont la rationalité ne soit pas rejetée au seuil de 5\% sont celles à l'horizon courant. Cependant, l'hypothèse nulle d'une prévision non biaisée est systématiquement rejetée au seuil de $5 \%$.

Les conclusions auxquelles nous arrivons sont à considérer avec prudence car la faible taille de notre échantillon (une quarantaine d'observations) remet en cause la robustesse de nos résultats. De plus l'utilisation de prévisions Consensus pose de nombreux problèmes. On peut discuter du choix des organismes retenus (étendre le choix du panel d'instituts interrogés n'améliorera la précision finale qu'à la condition que l'espace d'information mobilisé soit partiellement nouveau) ainsi que des pondérations à leur donner dans le calcul d'une prévision moyenne. D'autre part, le biais d'agrégation reste une des questions les plus discutées. Ainsi, il a été montré (Bonham et Cohen [2001] proposent un bon résumé technique de cette littérature théorique) que l'hypothèse de rationalité peut être retenue alors que les prévisions individuelles ne sont pas rationnelles (et inversement). Par ailleurs, ces affirmations théoriques ont été mises en exergue de manière empirique encore récemment par Dovern et Weisser [2011].

\footnotetext{
${ }^{9}$ L'article de Doz [1993] propose une très bonne synthèse.
} 
La suite de notre analyse se focalise donc sur des prévisions individuelles afin de s'affranchir de la possibilité d'un biais d'agrégation. Cependant, les données désagrégées publiées par le Consensus Economics ne portent que sur des prévisions de croissance dont la cible est soit la croissance de l'année en cours, soit la croissance de l'année à venir.

\section{Prévoir le taux de croissance pour l'année à venir (fixed-event forecasts)}

Nous travaillons maintenant avec des prévisions dont la cible, l'événement à prévoir, est fixé puisqu'il s'agit du taux de croissance pour l'année à venir. Mais les prévisions peuvent être émises à différentes périodes de l'année et peuvent être révisées. Nous éliminons de notre étude les prévisions pour l'année en cours car la rapidité avec laquelle sont exploitées les informations conjoncturelles devient un élément fondamental de la précision de ces prévisions. En revanche les prévisions pour l'année à venir sont des prévisions au sens strict qui ne peuvent s'appuyer sur aucune information conjoncturelle, même partielle.

\section{Les données}

Pour la période retenue (1999-2009) nous disposons de cinq sources d'information sur les prévisions de croissance pour l'année à venir : le Consensus Economics, le FMI, l'OCDE, la Commission Européenne et les prévisions du gouvernement issues du projet de loi de finance.

\section{Graphique 3 : Prévisions de croissance faites au mois de l'année N pour l'année N+1}

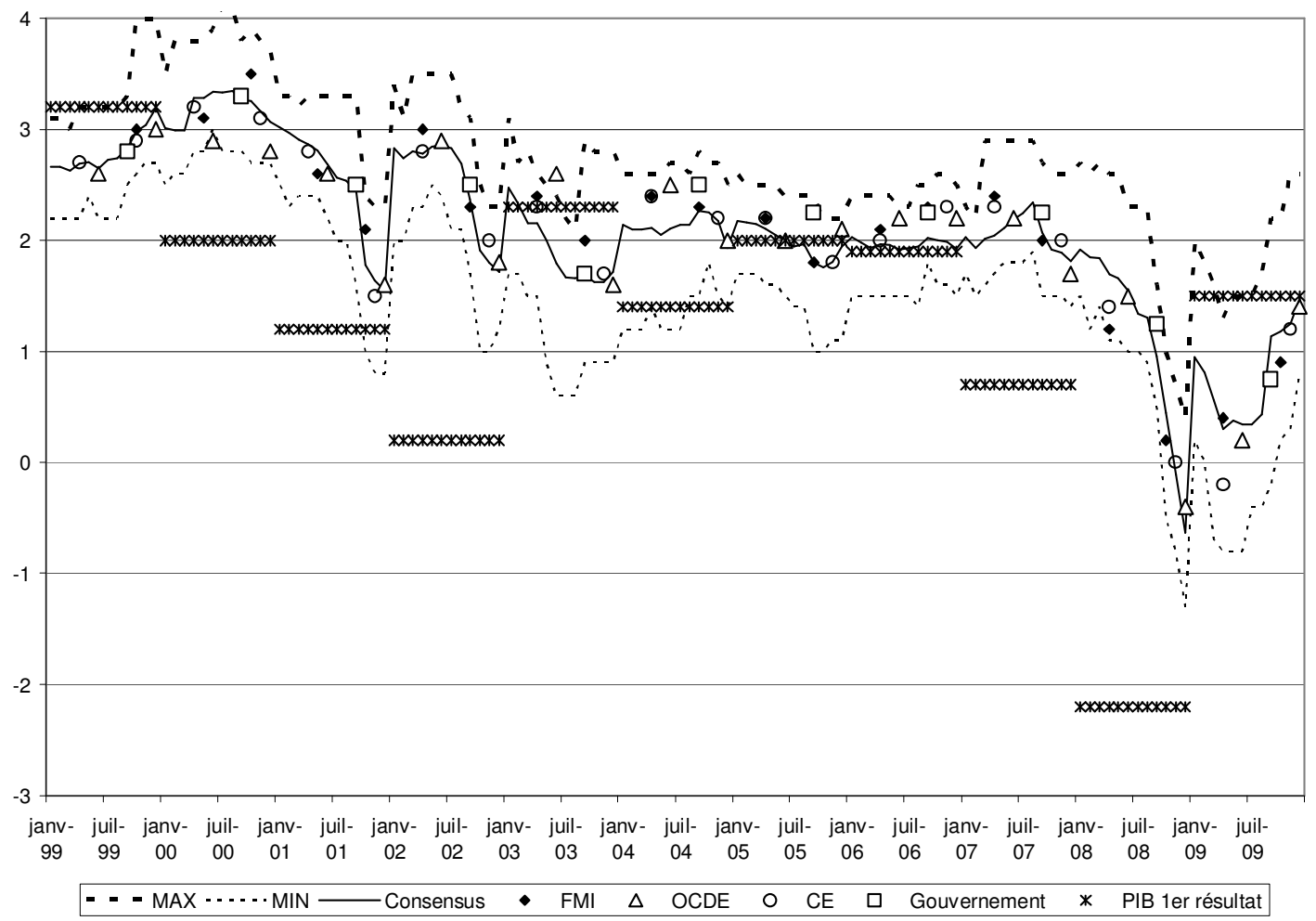

Source : Consensus Economics, Insee, FMI, OCDE, Commission Européenne, projet de lois de finance du gouvernement. 
Le graphique 3 propose un résumé des données comprenant les prévisions ponctuelles du FMI, de l'OCDE, de la Commission Européenne, du gouvernement, la prévision Consensus (prévision moyenne des organismes interrogées par le Consensus Economics), la prévision maximum du panel, la prévision minimum, et le taux de croissance annuel réalisé mesuré par le PIB 1er résultat. Un premier fait saillant est la convergence des prévisions qui fluctuent dans une bande d'une épaisseur moyenne de 1.2 point de croissance. Ensuite la proximité des prévisions des organismes officiels avec la prévision Consensus est marquante. Enfin, les années de crise (2001, 2002, 2003, 2008, et 2009) sont particulièrement mal prévues. Il est frappant que la prévision Consensus n'envisage la possibilité d'une récession (pour l'année à venir) qu'à la fin de 2008, quand l'économie vient de plonger dans l'une des plus grandes crises modernes.

\section{Les données individuelles du Consensus Economics}

Un des éléments cruciaux dans la constitution d'une base de données concerne le choix de la période étudiée. Pour ce qui concerne les données recueillies mensuellement par Consensus Economics, il faut avoir à l'esprit que les institutions interrogées en 1989 ne sont plus les mêmes que celles de 2010. Certaines ont disparu du sondage avec le siècle (Banque d'Orsay, Banque Paribas, Deutsche Bank, etc.), d'autres se sont transformées (Elf Aquitaine deviendra Total fina ELF, alors que Natexis sera racheté par la Banque Populaire qui fusionnera avec Ixis CIB pour devenir Natixis), et de nouvelles sont apparues durant les années 2000 (UniCredit, Oddo Securities, Citigroup, etc.). Il faut donc faire un arbitrage entre la profondeur de la base et le nombre d'institutions que l'on peut supposer homogène sur la période. En retenant comme période d'étude les prévisions émises entre 1999 et 2009 (soit potentiellement 132 observations), nous pouvons identifier neuf institutions homogènes dans l'échantillon pour lesquelles nous disposons de suffisamment de données : BIPE conseil, BNP, COE-CCIP, Crédit Agricole, Elf-Total, GAMA, Natixis, OFCE, Société Générale. En éliminant du panel la moitié des organismes interrogés nous ne modifions qu'à la marge les moments d'ordre un et deux. Le coefficient de corrélation entre la prévision moyenne calculée avec tous les individus du panel et celle obtenue uniquement avec les neuf institutions sélectionnées est de 0.99. Celui comparant les variances est de 0.73 (c'est sur la prévision de 2003 que des différences significatives apparaissent).

Le tableau 3 propose quelques statistiques sur les prévisions de chaque institution. Les prévisions disponibles sont les prévisions qui ont été renseignées alors que les prévisions effectives sont les prévisions qui diffèrent de celles du mois précédent. Les organismes proposent chaque mois dans plus de $80 \%$ des cas des prévisions de croissance pour l'année à 
venir. Cependant pour des institutions comme BIPE, COE, Elf ou encore l'OFCE ces prévisions sont dans plus de la moitié des cas les mêmes que celles proposées le mois précédent sans que l'on sache s'il s'agit d'un simple report ou s'il n'est apparu aucune information nouvelle nécessitant une modification de la prévision.

Tableau 3 : Quelques statistiques sur les prévisions individuelles

\begin{tabular}{|c|c|c|c|c|c|c|c|c|c|}
\hline & 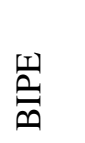 & $\stackrel{n}{Z_{n}}$ & 딩 & $=\frac{\dot{0}}{0}$ & $\frac{\Psi}{I I}$ & 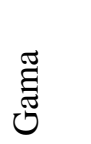 & 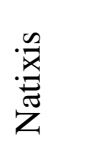 & 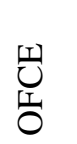 & 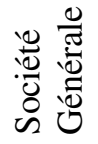 \\
\hline Part des prévisions disponibles & 0.86 & 0.93 & 0.79 & 0.87 & 0.85 & 0.87 & 0.95 & 0.86 & 0.90 \\
\hline Part des prévisions effectives & 0.36 & 0.53 & 0,29 & 0.41 & 0.36 & 0.66 & 0.51 & 0.27 & 0.51 \\
\hline $\begin{array}{l}\text { Ecart moyen de la prévision par } \\
\text { rapport au consensus }\end{array}$ & 0.06 & -0.21 & 0.11 & -0.02 & 0.13 & -0.06 & -0.03 & 0.19 & -0.14 \\
\hline $\begin{array}{l}\text { Part des prévisions supérieures à } \\
\text { celles du consensus }\end{array}$ & 0.53 & 0.21 & 0.55 & 0.49 & 0.75 & 0.37 & 0.47 & 0.75 & 0.18 \\
\hline Erreur de prévision moyenne & -.91 & -.68 & -.98 & -.97 & -.93 & -.79 & -.79 & -1.0 & -.72 \\
\hline $\begin{array}{l}\text { Part des erreurs de prévisions } \\
\text { négatives }\end{array}$ & 0.64 & 0.63 & 0.65 & 0.72 & 0.75 & 0.73 & 0.62 & 0.75 & 0.60 \\
\hline
\end{tabular}

La BNP a les prévisions qui s'écartent le plus en moyenne de la prévision Consensus, alors que celles du Crédit Agricole en sont les plus proches. L'OFCE produit les prévisions les plus optimistes en moyenne alors que la BNP est la plus pessimiste. L'examen de l'erreur moyenne de prévision fait apparaître un biais d'optimisme pour tous les organismes. Dans plus des deux tiers des cas, les erreurs de prévision sont négatives.

\section{Propriétés statistiques des prévisions publiées par le Consensus Economics}

Il existe une littérature spécifique pour l'analyse des propriétés des prévisions portant sur des événements à horizons fixés (fixed-event forecasts). Nordhaus [1987] en donne les bases pour des prévisions émises par un organisme unique, tandis que Davies et Lahiri [1995] étendent la méthode à plusieurs organismes (pooling) en utilisant les panels à trois dimensions: l'organisme qui établit les prévisions (noté i), la date de l'événement à prévoir (notée t), et l'écart entre la date de l'événement à prévoir et la date d'émission de la prévision (noté $h$ ). Le point de départ de toute analyse est la décomposition de l'erreur de prévision en trois composantes indépendantes :

$$
e_{i, t, h} \equiv R_{t}-P_{i, t, h}=\phi_{i}+\lambda_{t, h}+\varepsilon_{i, t, h} .
$$

La première composante de l'erreur, $\phi_{i}$, est le biais individuel de l'institut i. La second composante, $\lambda_{t, h}$ est commune à tous les prévisionnistes et reflète l'occurrence de chocs macroéconomiques. La troisième composante $\varepsilon_{i, t, h}$ est l'erreur inhérente à tout exercice de prospective. Ces trois composantes sont alors estimées de la façon suivante : 
$\hat{\phi}_{i}=\frac{1}{T H} \sum_{t=1}^{T} \sum_{h=13}^{H=24}\left(R_{t}-P_{i, t, h}\right) ; \quad \hat{\lambda}_{t, h}=\frac{1}{N} \sum_{i=1}^{N=9}\left(R_{t}-P_{i, t, h}-\hat{\phi}_{i}\right) ; \quad \hat{\varepsilon}_{i, t, h}=R_{t}-P_{i, t, h}-\hat{\phi}_{i}-\hat{\lambda}_{t, h}$

Un traitement préalable des données manquantes est nécessaire avant de décomposer les erreurs de prévisions. Nous suivons Davies et Lahiri (1995) qui proposent que chaque case individuelle manquante entourée de deux cases renseignées dont la valeur est identique reçoive la même valeur que ces deux cases. Dans le cas où les valeurs sont différentes, nous attribuons à la valeur manquante la dernière prévision émise. Ceci revient à supposer que le conjoncturiste n'a pas renseigné cette période car il n'avait pas de nouvelles prévisions à proposer. Enfin, si l'organisme n'a pas encore établi de prévision pour l'événement, aucune valeur n'est attribuée.

Tableau 4 : Biais des prévisions individuelles

\begin{tabular}{|c|c|c|c|c|c|c|c|c|c|}
\hline & 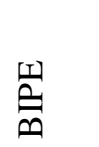 & $\sum_{m}$ & ن & 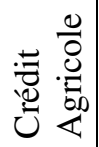 & $\frac{4}{I I}$ & 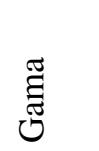 & 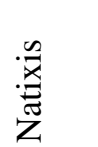 & $\begin{array}{l}\text { Uు } \\
\text { Uు }\end{array}$ & 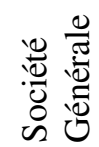 \\
\hline Estimation du biais individuel & -0.94 & -0.69 & -0.98 & -0.87 & -1.01 & -0.76 & -0.84 & -1.02 & -0.73 \\
\hline P. Value du test de biais & .006 & .027 & .004 & .010 & .003 & .016 & .015 & .008 & .024 \\
\hline
\end{tabular}

Le tableau 4 propose des statistiques relatives au test de biais des prévisions mené suivant la méthode de Davies et Lahiri (1995). Les écarts constatés sur les valeurs du biais individuel entre les tableaux 3 et 4 s'expliquent par le traitement des valeurs manquantes. Quelle que soit l'institution considérée, nous ne pouvons rejeter l'hypothèse d'un biais au seuil de $5 \%$. Le constat établi dans la section 1 d'un biais d'optimisme des prévisions est donc confirmé.

Pour finir, nous proposons d'analyser l'information apportée par le désaccord des neuf organismes $^{10}$. Pour cela, nous utilisons la régression de Döpke et Fritsche (2006) :

$$
\ln \left(\sigma_{t}\right)=\beta_{0}+\beta_{1} D_{t-1}^{\text {Contraction }}+\beta_{2} D_{t}^{\text {Contraction }}+\beta_{3} D_{t+1}^{\text {Contraction }}+\beta_{4} \text { Trend }+u_{t}
$$

où $\ln \left(\sigma_{t}\right)$, le logarithme de l'écart type des prévisions des organismes, est considérée comme une proxy du désaccord. La variable dummy vaut 0 durant les phases d'expansion et 1 durant les phases de contraction, phases obtenues à partir des datations classique du cycle de la France calculé par l'institut indépendant ECRI (Economic Cycle Research Institut).

Le tableau 5 donne les valeurs des coefficients estimés qui sont du même ordre de grandeur que celles trouvées par Dopke et Fritsche [2006] dans le cas de l'Allemagne. Cependant dans notre cas la variable dummy n'est pas significative en t. L'interprétation des résultats suggère que le désaccord s'accroit avant la contraction du cycle et décroit après.

\footnotetext{
${ }^{10}$ Voir Pesaran et Weale (2006 p749-750) pour une revue de la littérature concernant l'étude du désaccord entre un ensemble d'analystes.
} 
Tableau 5 : Estimation de l'équation (2)

\begin{tabular}{|l|c|c|c|c|c|}
\hline & $\beta_{0}$ & $\beta_{1}$ & $\beta_{2}$ & $\beta_{3}$ & $\beta_{4}$ \\
\hline Valeur du coefficient & -1.73 & 0.32 & 0.19 & -0.27 & 0.004 \\
\hline Valeur du rapport de Student & -15.64 & 2.42 & 1.19 & -1.87 & 2.05 \\
\hline
\end{tabular}

\section{Conclusion}

Il est loin le temps des trente glorieuses où le taux de croissance moyen oscillait autour de $5 \%$ avec un écart type de $1.5 \%$. Les valeurs observées de la croissance (avec un maximum de $8 \%$ en 1950 et un minimum de $2.6 \%$ en 1959) étaient dans l'intervalle de confiance au seuil de $5 \%$ issu d'une loi normale : [+8\%; +2\%]. Depuis la crise de 1974, le taux de croissance moyen (sur la période 1975-2010) n'est plus que de $2.1 \%$, pour un écart type du même ordre (1.5\%). La récession fait maintenant partie de l'intervalle de confiance $[5.1 \% ;-0.9 \%$ et ce phénomène a pu être observé en $1993(-0.7 \%), 2008(-0.1 \%)$ et 2009 (-2.7\%). Pourtant il semble que les instituts de conjoncture n'aient pas encore pris toute la mesure de ce changement en émettant des prévisions beaucoup trop optimistes.

Il est d'ailleurs frappant que les experts ne prennent jamais le risque d'envisager une récession, sauf dans le cas où celle-ci est déjà installée. La boutade de Warren Buffet «les prévisions vous en disent beaucoup sur ceux qui les font, elles ne vous disent rien sur l'avenir » renvoie aux travaux sur la fonction de perte des experts. Patton et Timmermann [2004] montrent de manière empirique que l'hypothèse de rationalité est moins souvent rejetée si l'on considère que les experts ont une fonction de perte non symétrique. Cela laisse entendre que certains analystes attribueraient un coût supérieur au fait de sous-évaluer la valeur d'une variable à prévoir. Des raisons politiques peuvent aussi être envisagées comme par exemple la volonté d'induire une certaine politique économique ou bien encore l'intention d'obtenir une certaine renommée en s'écartant volontairement de l'ensemble des autres analystes. Toutefois, comme l'expliquent Batchelor [2007] et Dovern et Weisser [2011], ces raisons sont peu probables dans le cas de prévisions Consensus.

L'importance du biais des prévisions de croissance est essentielle pour les finances publiques. La révision de la prévision croissance en France pour 2012 (de 1.75\% à 1\%) pendant l'examen du projet de loi de finance a imposé environ 8 milliards d'euros d'efforts supplémentaires. L'ordre de grandeur de la révision ( 0.75 point de croissance) n'est pas exceptionnelle. L'erreur de prévision moyenne (par rapport au PIB définitif) sur la période 1999-2008 est de 0.51 point si on considère les prévisions de la loi de finance, de 0.33 point avec les prévisions Consensus 
de septembre, de 0.44 point avec les prévisions de l'OCDE et du FMI. Des propositions politiques fleurissent pour réduire le coût du biais d'optimisme de la prévision associé à la loi de finance, comme par exemple de confier cette prévision à un organisme indépendant ou encore ne retenir que la prévision la plus pessimiste d'un panel d'instituts.

Une autre solution serait de ne plus se limiter à la seule information apportée par la prévision émise (la valeur centrale de la prévision) et de prendre aussi en compte les probabilités d'occurrence des événements ${ }^{11}$. En effet, un expert peut émettre une prévision de croissance positive tout en considérant qu'une récession fait partie des scénarios envisageables. C'est ainsi que la Banque Centrale d'Angleterre fournit depuis 1996 une prévision en densité de l'inflation appelée fan chart. En France, l'Insee publie depuis 2008 un graphique des risques associés à sa prévision PIB dans sa note de conjoncture. Cornec [2010] propose pour les prévisions de la croissance française un fan chart conditionnel, semi-paramétrique et reproductible, qui permet de lier la précision de la prévision à la conjoncture économique.

\section{Bibliographie}

AGER P., M. KAPPLER et S. OSTERLOH [2009], "The accuracy and efficiency of the consensus forecasts: a further application and extension of the pooled approach", International Journal of Forecasting, 25, p. 167-181.

BATCHELOR R. [2007], "Bias in macroeconomic forecasts", International Journal of Forecasting, 23, p. 189-203.

BESSEC M. [2010], " Etalonnages du taux de croissance du PIB français sur la base des enquêtes de conjoncture ", Economie et Prévision, 193, p. 77-99.

BONHAM C. et R. COHEN [2001], "To Aggregate, Pool, or Neither: Testing the RationalExpectations Hypothesis Using Survey Data", Journal of Business \& Economic Statistics, 19, p. 278-91.

CHARPIN J.M. [2010], "Statistiques : les voies de la confiance", Revue économique, 61, p. 371-393.

CHARPIN F. [2011], " Réévaluation des modèles d'estimation précoce de la croissance", Revue de l'OFCE, 118, p. 129-142.

CORNEC M. [2010], "Constructing a conditional GDP fan chart with an application to French business survey data", Workshop DGECFIN, Bruxelles, 16 November 2010.

DAVIES A et K. LAHIRI [1995], "A new framework for analysing survey forecasts using three-dimensional panel data", Journal of Econometrics, 68, p. 205-227.

\footnotetext{
${ }^{11}$ Pour une revue de la littérature, voir Tay et Wallis [2000].
} 
DOPKE J. et U. FRITSCHE [2006], "When do forecasters disagree? An assessment of German growth and inflation forecast dispersion", International Journal of Forecasting, 22, p. $125-135$.

DOVERN J., U. FRITSCHE et J. SLACALEK [2009], "Disagreement among forecasters in G7 countries", European Central Bank, Working paper n¹082.

DOVERN J. et J. WEISSER [2011], "Accuracy, unbiasedness and efficiency of professional macroeconomic forecasts: An empirical comparison for the G7", International Journal of Forecasting, 27, p. 452-465.

DOZ C. [1993], " Note sur les tests de rationalité des prévisions", Economie et Prévision, 108, p. 129-133.

GRANGER C.W.J. [1969], "Prediction with a Generalized Cost of Error Function. Operational Research Quarterly, 20, p. 199-207

ISIKLAR G., K. LAHIRI et P. LOUNGANI [2006], "How quickly do forecasters incorporate news? Evidence from cross-country surveys", Journal of Applied Econometrics, $21,703-725$.

LOUNGANI P., H. STECKLER et N. TAMIRISA [2009], "Cross-Country Evidence on Forecasting Turning Points: Consensus and Disagreement", IMF Working Paper.

MINCER J. et V. ZARNOWITZ [1969], "The evaluation of economic forecasts", In J. Mincer (Ed.), Economic forecasts and expectations. New York: National Bureau of Economic Research, p. 1-46.

NORDHAUS W. [1987], "Forecasting Efficiency: Concepts and Applications," The Review of Economics and Statistics, 69, p. 667-674.

PATTON A. et A. TIMMERMANN [2011], "Forecast Rationality Tests Based on MultiHorizon Bounds", CEPR Discussion Papers 8194, C.E.P.R. Discussion Papers.

PATTON A. et A. TIMMERMANN [2004], "Properties of optimal forecasts under asymmetric loss and nonlinearity", Mimeo, London School of Economics and Department of Economics, University of California, San Diego.

PESARAN H. et M. WEALE [2006], "Survey Expectations," Handbook of Economic Forecasting, Volume 1, p. 715-776

TAY S. et F. WALLIS [2000], "Density forecasting: a survey", Journal of Forecasting, 19, 235-254.

TIMMERMANN A. [2007], "An evaluation of the World Economic Outlook forecasts", IMF Staff Papers, 54, p. 1-33. 